\title{
MASSIVE PERICARDIAL HEMATOMA SIMULATING CONSTRICTIVE PERICARDITIS: A COMPLICATION OF RADIOFREQUENCY CATHETER ABLATION
}

\author{
Anastasios K. Konstantakos, MD, ${ }^{\text {a }}$ Robert C. Gilkeson, MD, ${ }^{\mathrm{b}}$ Frank V. Brozovich, MD, PhD, ${ }^{\mathrm{c}}$ and Jai H. Lee, MD, \\ Cleveland, Ohio
}

Radiofrequency catheter ablation (RFCA) has become an increasingly popular technique for nonpharmacologic treatment of various cardiac arrhythmias because of its efficacy and low complication rate. ${ }^{1}$ This appears to be the first reported case of an occult myocardial perforation induced by RFCA that led to a clinical presentation of constrictive pericarditis and right ventricular (RV) obstruction.

Clinical summary. A 54-year-old man with a longstanding history of atrial flutter (AF) was seen for elective evaluation of recurrent bouts of exertional dyspnea, worsening exercise tolerance, and occasional palpitations that had developed over a 3-year period. During this time he was diagnosed with constrictive pericarditis. He had a mild stroke 15 years ago and had been placed on a warfarin sodium (Coumadin) anticoagulation regimen since then. Approximately 3 years before this presentation he had an RFCA for attempted treatment of the AF. However, during electrophysiologic testing, AF could not be induced, and several radiofrequency energy lesions were placed in the lower right atrial area near the presumed origin of the AF circuit.

Because he continued to have episodes of symptomatic $\mathrm{AF}$, he was admitted electively for evaluation and possible RFCA of his AF. During this second attempt at RFCA, $\mathrm{AF}$ was not inducible. However, it was noted on fluoroscopy and subsequently confirmed by chest x-ray films that the patient had diffuse nodular epicardial and curvilinear pericardial calcifications anterior to the RV (Fig. 1). A subsequent echocardiogram showed a thickening around the RV that greatly reduced the RV chamber size. Cardiac catheterization revealed decreased RV and pulmonary artery pressures with a well-defined "dip and plateau" pattern of restrictive filling. Magnetic resonance imaging scans demonstrated a homogenous mass anterior to and compressing the RV (Fig. 2).

From the Division of Cardiothoracic Surgery, ${ }^{a}$ Department of Radiology, ${ }^{\mathrm{b}}$ Division of Cardiology, ${ }^{\mathrm{c}}$ Case Western Reserve University, University Hospitals of Cleveland, Cleveland, Ohio.

Received for publication Oct. 3, 1997; accepted for publication Oct. 10, 1997.

Address for reprints: Jain H. Lee, MD, Division of Cardiothoracic Surgery, Case Western Reserve University, University Hospitals of Cleveland, 11100 Euclid Ave., Cleveland, OH 44106.

J Thorac Cardiovasc Surg 1998;115:726-7

Copyright (C) 1998 by Mosby, Inc.

$0022-5223 / 98 \$ 5.00+0 \quad \mathbf{1 2 / 5 4 / 8 6 8 8 1}$
The patient was taken to the operating room for exploration through a median sternotomy. Operative dissection revealed a thickened pericardium with dense adhesions to the epicardium of the RV and left ventricle. A $12 \mathrm{~cm}$ calcified mass, which appeared to be an old hematoma, was noted adjacent to the diaphragmatic surface of the RV and extended laterally to the left ventricle. Dissection around this mass was accomplished, but because of patchy ingrowth into the epicardium, a small part of the RV myocardium was entered and was repaired. After resection of the mass, RV compression was relieved, and RV contractility had markedly improved.

Gross pathologic examination confirmed the presence of an old calcified hematoma. Histologic examination revealed an organizing thrombus with focal dystrophic calcifications. The patient's recovery was uneventful. He has a postoperative echocardiogram that showed complete resolution of the compression and markedly improved RV function. At follow-up 6 months postoperatively, the patient was well and reported no complaints.

Comment. The most likely cause of this hematoma is an occult myocardial perforation at the time of the first RFCA, which led to the slow accumulation of pericardial blood and subsequent organization and calcification over 3 years' duration. The patient's warfarin therapy also could have predisposed him to this low-grade, occult bleeding. Furthermore, it has been suggested that injured myocardium from RFCA therapy may be less resistant to rises in ventricular pressure. ${ }^{2}$ Thus it is quite possible that

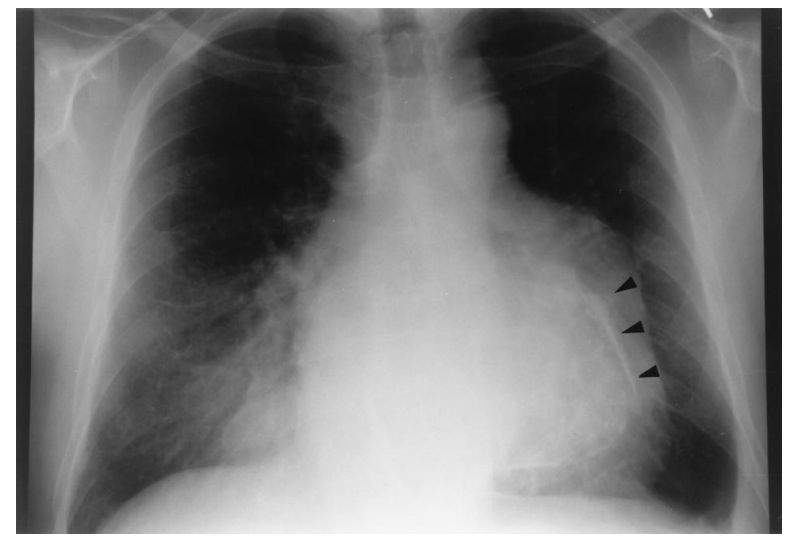

Fig. 1. Preoperative chest radiograph demonstrating thick curvilinear calcifications overlying the cardiac silhouette (arrowheads). Extensive, abnormal soft tissue opacity extends beyond these calcifications. 


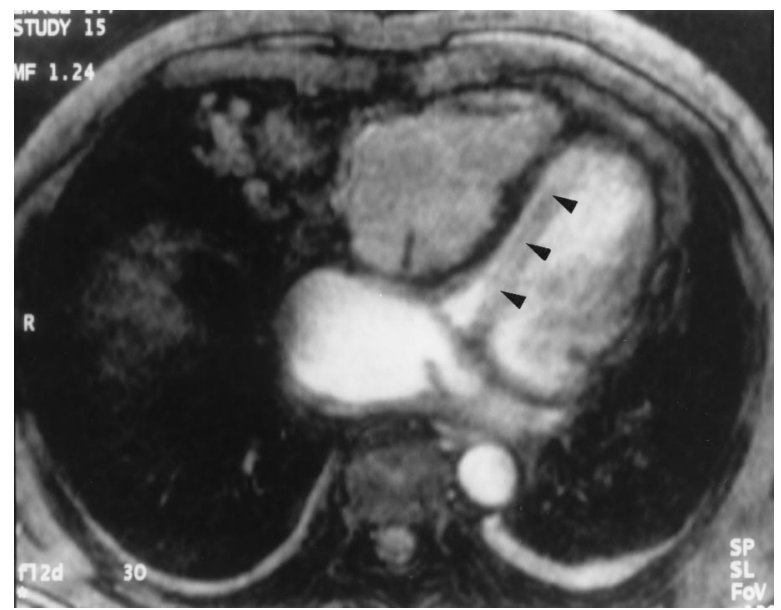

Fig. 2. Preoperative axial cine magnetic resonance image at the ventricular level demonstrating a pericardial/epicardial mass causing marked compromise of the right ventricular chamber (arrowheads).

a delayed rupture of the myocardium occurred sometime after RFCA therapy and led to a slow accumulation of hemopericardium.

Although we were not able to determine the exact site of the chamber perforated, rupture of the right atrium would have been unlikely. The immediate accumulation of blood within the pericardium owing to the inability of atrial tissue to locally contract and contain extravasation would have acutely manifested itself as pericardial tamponade.

The etiologic spectrum of constrictive pericarditis consists of postradiotherapy, postoperative, and postinfectious sequelae, as well as neoplasia, connective tissue disorders, and autoimmune disease. ${ }^{3}$ A persistent intrapericardial hematoma induces pericardial inflammation, leading to granulation tissue, adhesions, and finally constriction. ${ }^{4}$ Thus the possibility of an organized pericardial hematoma should not be overlooked in the differential diagnosis of constrictive pericarditis after RFCA therapy.

Definitive diagnosis and therapy of constricting pericardial masses depends on operative removal as illustrated in this report.

\section{REFERENCES}

1. Hindricks G. The multicenter European radiofrequency survey (MERFS): complications of radiofrequency catheter ablation of arrhythmias. Eur Heart J 1993;14:1644-53.

2. Kongsgaard E, Foestrster A, Aass H, Madsen S, Amie JP. Effects of combined radiofrequency and direct current energy catheter ablation on ventricular myocardium in pigs. Eur Heart J 1995;16:514-20.

3. Cameron J, Oesterle SN, Baldwin JC, Hancock EW. The etiologic spectrum of constrictive pericarditis. Am Heart J 1987;113:354-60.

4. Brown DL, Ivey TD. Giant organized pericardial hematoma producing constrictive pericarditis: a case report and review of the literature. J Trauma 1996;41:558-60. 